\title{
How plant-animal interactions signal new insights in communication
}

\author{
H. Martin Schaefer ${ }^{1}$, Veronika Schaefer ${ }^{1}$ and Douglas J. Levey ${ }^{2}$ \\ ${ }^{1}$ Institute for Biology 1, Albert Ludwigs-Universität, Freiburg, Hauptstr. 1, 79104 Freiburg, Germany \\ ${ }^{2}$ University of Florida, Department of Zoology, PO 118525, Gainesville, FL 32611-8525, USA
}

\begin{abstract}
Fundamental concepts about the diversity and evolution of signals have been developed mainly in the context of sexual selection. Here, we review the functional ecology of signals in a different context, that of plant-animal interactions. The visual signals of fruits and flowers are relatively constant and clear in the message that they convey. Thus, plant signals present a particularly suitable, albeit overlooked, system with which to study signal evolution. Plant signals are aimed at multiple receivers, mutualists and antagonists, and understanding how fruit and nectar consumers perceive plant signals provides the key with which to evaluate those signal components that work efficiently for a given consumer. Recent developments in the field of plant-animal communication shed new light on fundamental concepts in signal theory such as mimicry, honest signalling, private communication channels and the sensory drive hypothesis.
\end{abstract}

Communication is fraught with challenges. Senders and receivers often differ in their attention to signals and in their intentions based on those signals. Thus, even though listeners alert to a precisely transmitted signal receive it clearly, they might be unable to assess its reliability accurately. Imagine, then, the difficulty of eavesdropping as a human on signals conveyed by other organisms and interpreting them in an ecological or evolutionary context. Assumptions abound and it is not surprising that concepts in signal theory are still contentious [1].

The evolution of signals has attracted much recent attention, uniting such disparate disciplines as biochemistry, cognition and functional ecology [1]. Although there are many types of signal [2,3], most attention has focussed on epigamic signals; that is, signals that are used to communicate between sexes and which are often the result of sexual selection. The inherent conflict between male and female reproductive strategies complicates the assessment of epigamic signals. Moreover, they are often complex, involving a combination of different sensory modes, such as colour patterns, sound and movement. Finally, different individuals might advertise different qualities and pursue different strategies in sending and reacting to signals [4].

Corresponding author: H. Martin Schaefer (martin.schaefer@biologie.unifreiburg.de).

Available online 1 September 2004
Here, we suggest that the focus on epigamic signals has caused other less dynamic signalling systems to be overlooked. These systems might enable unusually direct tests of some aspects of signal theory and we call attention to the communication between plant reproductive structures (i.e. fruits and flowers) and the animals that react to them. Signals from plants to animals are relatively constant in space and time without, for example, the option for modifying them in the presence of predators, and they are often less complex and dynamic compared with animal signals. Furthermore, they often lack a behavioural component, are simple in structure and are displayed against an unchanging background (Table 1). Equally important, the intentions of the sender are as unambiguous and context independent as the interests of the receivers in that signal. Animals attend to the signals to gain a nutritional reward as in prey-predator systems. But, unlike most prey, plants signal to attract consumers who are mutualists (i.e. seed dispersers or pollinators). For example, changes in the nutritional reward of fruit during ripening are paralleled by obvious changes in the signal (Figure 1a). Consequently, the 'honesty' of the signal that advertises the reward is relatively easy to assess, as are the fitness consequences of reacting to the signal.

We begin by contrasting the evolutionary ecology of signal design in fleshy fruits and flowers and then review the potential for signals between plant reproductive parts and animals to provide new insights into three key issues of signal theory:

(i) Multiple receivers. How do disparate types of receiver interact with signal design and to what extent are signals either general in design or targeted to particular sensory capabilities of mutualists? Are different signal components designed to attract mutualists and/or to deter antagonists?

(ii) Honest signalling. Do plants convey accurate information about nutritional rewards in fruits and flowers? How common is 'cheating' (i.e. mimicry) by the sender?

(iii) SENSORY DRIVE (see Glossary). Are the signals used by plants to attract mutualists mirrored by the intraspecific signals used by those mutualists?

Although plants signal the presence of fruits and flowers through both olfactory and visual channels (Table 2), we focus on visual channels because the literature on such signals [5-8] and on the perception 


\section{Glossary}

Dichromatic: requires two spectral receptor types and associated neural mechanisms.

Photosensitivity: the probability that a photon of a given wavelength elicits a neural response.

Sensory drive (model): (a model focusing on) how communication systems adapt to local environments and encompassing all steps from signal generation to perception.

Sensory exploitation: a model predicting that properties of the sensory system shape perception and affect (mate) preferences. Traits that are most effective in stimulating the sensory system are preferred.

Tetrachromatic: requires four spectral receptor types and associated neural mechanisms.

Trichromatic: requires three spectral receptor types and associated neural mechanisms.

and colour processing of consumers [9-13] has increased considerably during recent years. Moreover, visual signals are usually more effective than are olfactory signals for long-distance signalling owing to the considerable influence that the environment has on the dispersal of pheromones $[14,15]$.

\section{The comparative ecology of fruit and flower signals}

Flowers and fruits share the same message ('visit me') and the same pigments (anthocyanins and carotenoids), but differ in design. Fruits are usually unicoloured, whereas flowers are more variable in colour pattern and shape. Much of this difference is probably attributable to genetic constraints, because most fruits develop from a single flower structure (the ovary wall develops into the pericarp of the fruit), whereas flowers comprise several ontogenetically differentiated structures that serve multiple purposes (e.g. signalling to pollinators, nectar production and reproduction). The fundamental difference in the signal content between fruits and flowers is that fruits constitute a signal and a reward, whereas both are essentially decoupled in flowers: flowers signal the presence of rewards through the corolla or other floral parts but these parts are not in themselves rewarding. The decoupling means that pollinators cannot reliably assess flower rewards from a distance. We suggest that this decoupling triggered the evolution of a behavioural trait in pollinators that is less pronounced in seed dispersers: flower constancy (i.e. the tendency to restrict foraging bouts to one or few species with a known reward [16]). Plants benefit from flower constancy by reducing the deposition of heterospecific pollen and increasing that of conspecific pollen [16]. Flower constancy is facilitated by the flowers being easily recognizable, thus favouring the evolution of high signal diversity, especially among rare or dispersed species [5].

With respect to seed dispersal, plants do not benefit as much from consumer constancy, because a frugivore restricting foraging bouts to a single species would inevitably increase the probability of seeds being deposited under conspecifics, where mortality is disproportionately high [17]. Thus, the evolution of fruit signals was probably driven towards convergence on the most successful signals. Indeed, 50-70\% of bird-dispersed temperate, and a similar proportion of bird-dispersed tropical, fruits are either red or black [18]. Calculating the Euclidean distance between fruit and background spectra shows that these colours contrast most with the surrounding leaves and bark [6]. However, some plants display accessory

Table 1. Comparison of visual signal characteristics in animal-animal and plant-animal communication ${ }^{a}$

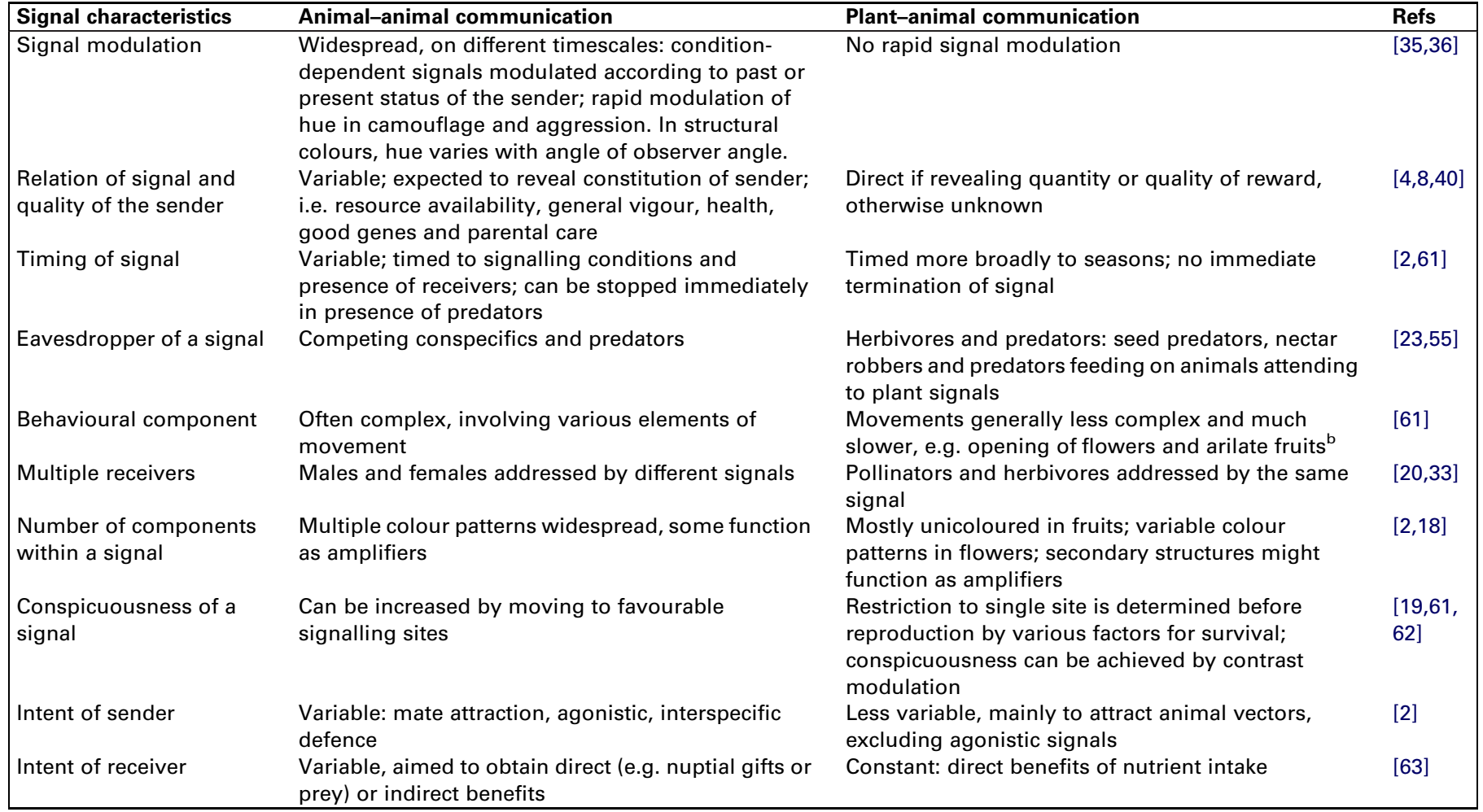

${ }^{a}$ Only the first three qualities denote absolute differences, whereas the other characteristics vary within both communication system and, therefore, reflect differences in degree of signal characteristics.

${ }^{b}$ Arilate fruits are those that are covered by a husk or capsule that opens to reveal the fleshy fruit and seeds. 

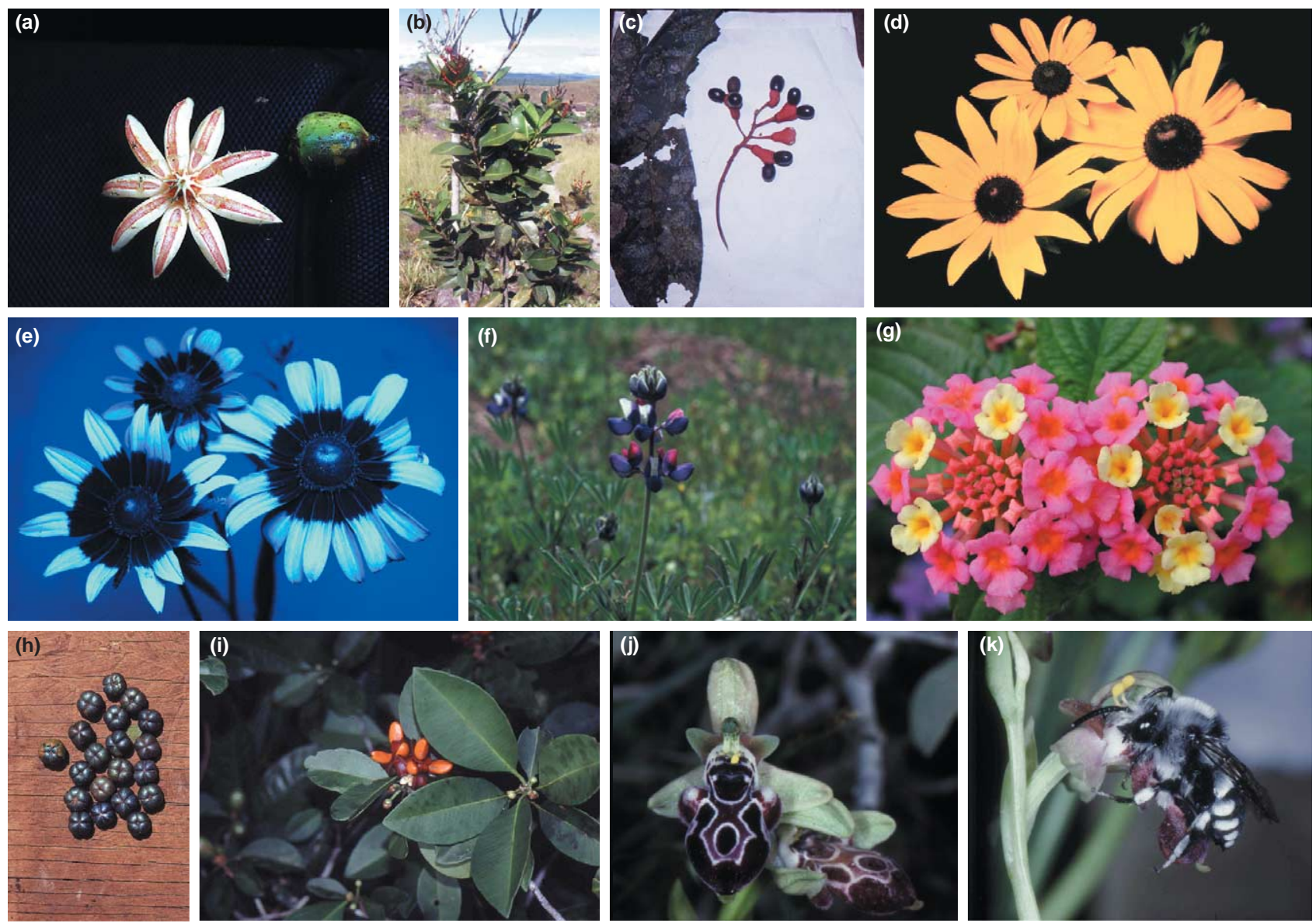

Figure 1. How plants signal fruits and flowers to animals that forage on them. (a) Ripe (left) and unripe (right) fruits of attorney Clusia sp. demonstrating how plant signals change with increasing nutritional contents. Unripe fruits containing not yet viable seeds do not aim to be eaten. They are generally green and cryptic against the foliage. As fruits ripen, the nutritional fruit content increases and this is paralleled by a dramatic change in the signal when fruits open. (b,c) Ouratea Ouratea sp. The red coloured stems of the infructescence probably function as amplifiers of the signal to increase the conspicuousness of the black fruits. In general, such amplifiers are often red and accompany black fruits. (d,e) Floral images in the visible and UV light of the flowers of the black-eyed Susan Hypericum calycinum. Dearomatized isoprenyl phloroglucinols absorb UV causing the dark centre of the flowers under UV light. These pigments function not only to attract pollinators but also as a feeding deterrent for herbivores documenting a dual signal function in these pigments. (f) The sky lupine Lupinus nanus signals honestly to pollinators: the banner spot of the petals changes from white to red after pollination has occurred. These non-rewarding flowers increase the attractiveness of the display while increasing the foraging efficiency of pollinators. (g) In red sage Lantana camara, floral colour indicates nectar availability: yellow flowers offer more nectar than pink flowers. (h) The mimetic fruits of margaritaria Margaritaria sp. offer no reward to the consumer. (i) In the Venezuelan rainforest, the orange-yellow hues in fruits signal high protein contents to the consumer, as in this Tovomita sp. Birds, being the main consumer group, prefer fruits with high protein contents. (j.k) Orchids of the genus Ophrys eavesdrop on the signals of their pollinators. They do not offer a reward for pollination but mimic virgin females to induce pollination. Flowers of Ophrys kotschyi show the typical white and dark markings of their pollinator, the bee Melecta tuberculata. Reproduced with permission from H.M. Schaefer $(\mathrm{a}-\mathrm{c}, \mathrm{h}, \mathrm{i})$, Tom Eisner $(\mathrm{d}, \mathrm{e})$, Martha Weiss $(\mathrm{f}, \mathrm{g})$ and Claudia Gack $(\mathrm{j}, \mathrm{k})$.

structures (e.g. coloured bracts and stems) together with the fruits or flowers that probably function as amplifiers, increasing the detectability of the entire display [19] (Figure $1 b, c)$.

\section{Multiple receivers and signal matching}

Multiple receivers, including mutualists and antagonists, can perceive most visual signals. This is not trivial, given that many studies of signal evolution focus on only one type of receiver. For example, in sexual selection, males and females might exert different selection pressures on a signal, thus representing independent receivers [20]. Likewise, cryptic and aposematic colouration of prey are directed towards multiple receivers, yet are rarely interpreted from the perspectives of multiple predators [21,22]. We argue that the evolution of signals is best understood by considering all receivers, especially those with disparate ecologies. Selection pressures by different receivers might result in directional as well as disruptive signal evolution [23,24]. In the wild radish Raphanus sativus, pollinators prefer anthocyanin-recessive white floral colour morphs, but frequencies of anthocyanin-dominant pink morphs remain constant over time. How is this polymorphism maintained? Anthocyanin-recessive flowers suffer more herbivore damage than do the anthocyanin-dominant flowers because the latter are more strongly defended by secondary compounds such as glucosinolates. The diverging selection pressures of pollinators and herbivores result in an evolutionary stable flower colour polymorphism [23]. A similar influence of multiple receivers on signal design occurs in the Andean monkeyflower Mimulus luteus, where selection for nectar guides exerted by insect pollinators and by hummingbirds probably leads to the disruptive selection of nectar guide size and shape [24].

Given that intended receivers, such as pollinators, are beneficial to the plant, and unintended receivers, such as 
Table 2. Vision and scent as two signalling modes in plant-animal communication

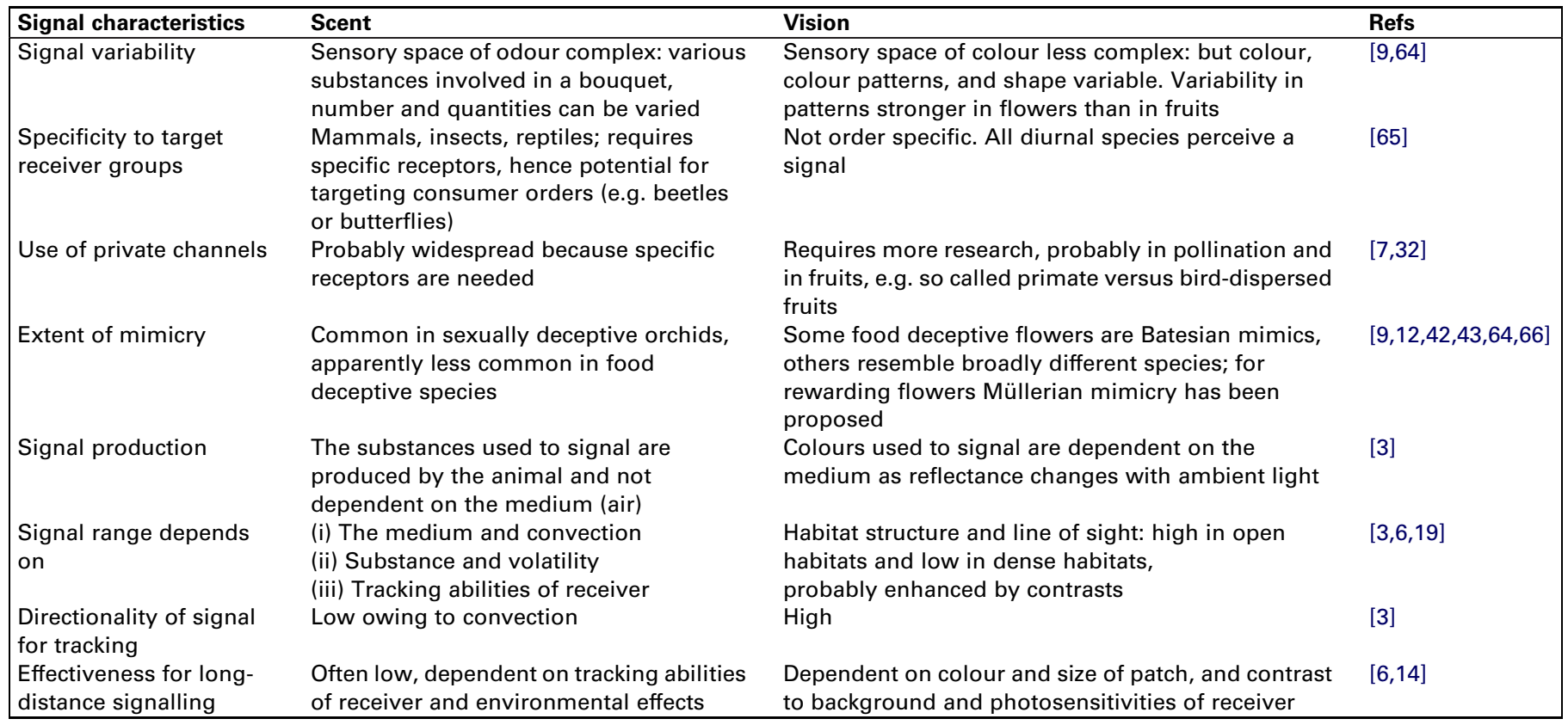

herbivores, are harmful, signal design in non-polymorphic species is probably a tradeoff between high conspicuousness to intended receivers and relative inconspicuousness to unintended receivers [2]. This tradeoff might be more pronounced in plant signals than in animal signals because plants cannot rely on mobility or behaviour to escape unintended receivers. Therefore, they are forced to mitigate the conflict solely through signal design. The dilemma of how to make a visual signal simultaneously conspicuous to some receivers and inconspicuous to others can be solved if receivers differ in their PHOTOSENSitivities. Conceivably, a signal can be matched to the portion of the spectrum in which photosensitivities are high in mutualists and low in antagonists or vice versa. The extent to which such 'private channels' are used to convey information is a fundamental issue in the ongoing debate about whether signals are adaptively fine-tuned versus constrained or arbitrary.

Signals associated with fruits and flowers are also well suited for our assessment of the frequency of private communication channels, because their receivers often differ in visual capacities. Pollinators and seed dispersers comprise TETRACHROMATIC birds, TRICHROMATIC insects, and tri- and DICHROMATIC mammals [13,25-27]. Although the ability to discriminate colour largely depends on the number of retinal receptor types [28], there are other important determinants. The visible spectrum differs: from $\sim 310-700 \mathrm{~nm}$ in passerine birds and some insects, to $\sim 350-700$ in most non-passerines birds, to $\sim 310-580 \mathrm{~nm}$ in many other insects, and to $400-700 \mathrm{~nm}$ in mammals. The ability to discriminate colour also varies within each of these groups because the maximum sensitivities of the receptor types differ among taxa [29]. We suggest that there is plenty of opportunity for targeting (or avoiding) particular consumer subsets, especially with red or UV signals, which are relatively inconspicuous to bees and mammals, respectively (Figure 2). Multiple receivers that impose differential selection pressures on plant traits are not confined to the broad division of mutualists and antagonists. Pollinators and seed disperser species can also differ in the degree to which they benefit plants. The three-wattled bellbird Procnias tricarunculata reliably deposits laurel Ocotea endresiana seeds in favourable germination sites, whereas other dispersers, such as guans and quetzals, do not [30]. Likewise, bumblebees Bombus spp. pollinate some flowers but rob nectar from hummingbird-pollinated flowers [31].

We hypothesize that plant signals in more specialized syndromes match the photosensitivities of the most effective dispersers or pollinators and are relatively inconspicuous to less effective groups; typical examples include differences in the colours of primate- versus birddispersed fruits [32]. The evolution of such 'private channels' does not require exclusive use, only moreefficient perception by the targeted mutualists. In other words, a private channel constitutes those signals that are better detected by the intended, rather than other, receivers. In sister species of Mimulus monkey flowers, allele substitution resulting in floral colour change induces a simultaneous switch in the pollinator spectrum. Bees visit almost exclusively pink flowers and hummingbirds almost exclusively red flowers. When flower colour is genetically switched, the types of pollinator shift in parallel [7], possibly demonstrating the use of 'private channels' in the communication between Mimulus and its pollinators.

Uncovering other examples of private channels will require embracing the notion that receivers influence plant signals, with disparate effects on plant fitness. In this respect, the classic idea of disperser and pollination syndromes might be outdated. Such syndromes might not be targeted solely at mutualists (as traditionally assumed), but might also be aimed at avoiding antagonists [23]. The most obvious example are UV-reflecting floral 


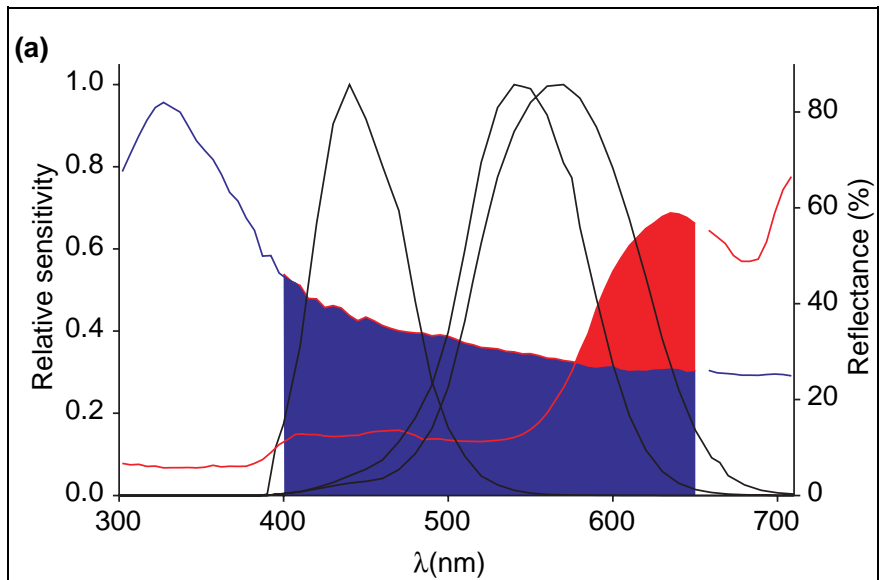

(b)

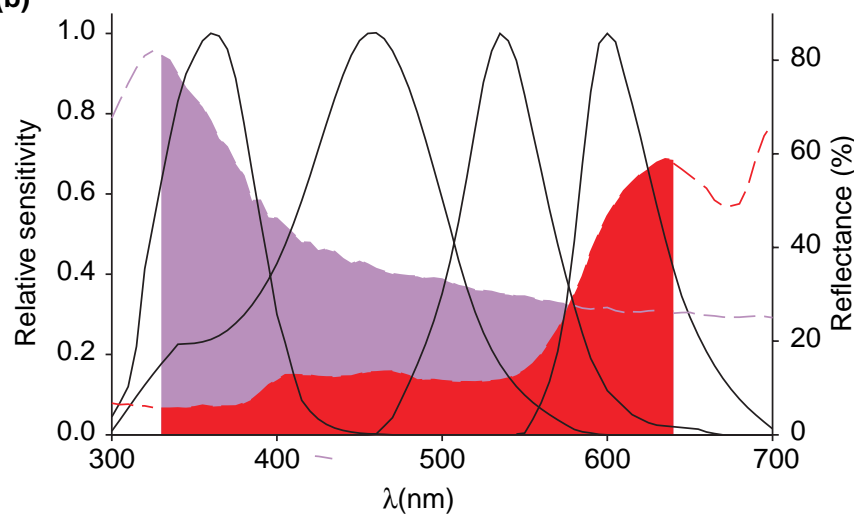

(c)

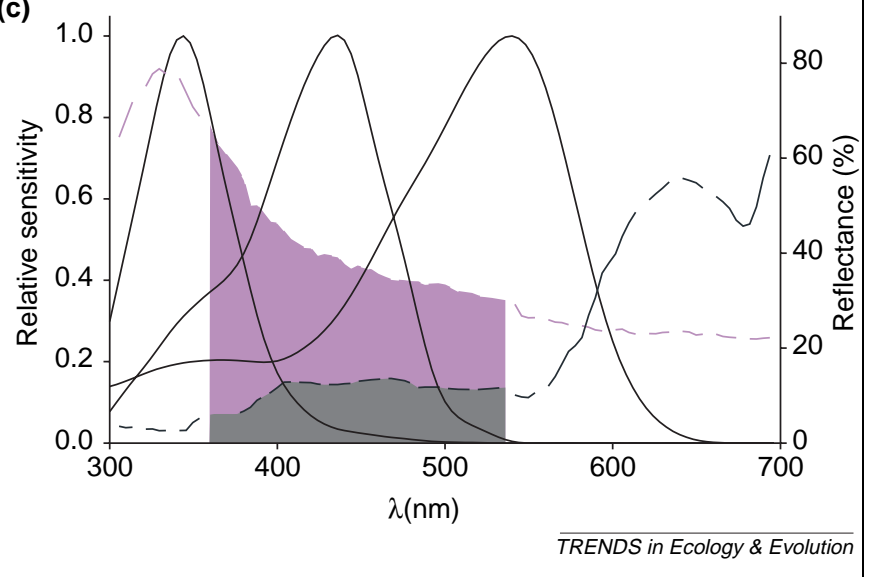

Figure 2. Spectra of a blueberry Vaccinium sp. (peak in the UV part) and willowleaf cotoneaster Cotoneaster salicifolia (peak in the human red part of the spectrum) according to the colour perception of humans (a), birds (b) and bees (c). The colours do not represent the actual colour sensation of each group but rather serve as an approximation to illustrate differences in visual abilities. Solid black lines denote the spectral sensitivities of the four (birds) and three receptor types in bees and humans based on physiological measurements and behavioural tests resulting in models of colour vision [25-27]. If at least two receptors are excited by photons of a given wavelength, wavelength discrimination is possible. Vertical lines denote this range for each group.

pigments (de-aromatized isoprenylated phloroglucinols), which are used to attract mutualists, but which also function simultaneously as chemical defence against herbivores (Figure 1d,e) [33]. Given that some of these pigments are also found in the leaves of these species, the signal function of attracting mutualists might be a pleiotropic effect of defence against herbivores [34].

\section{Honest signalling}

Although some male ornaments function as accurate indicators of male quality $[35,36]$, the general occurrence of such 'honest' signals remains controversial [37,38]. Conflicting reproductive strategies between, and behavioural flexibility within, the sexes [4] might constrain the evolution of honest sexual signals. These problems appear to be less pronounced in the communication system between plants and animals, rendering analyses of the relationship between signal design and reward in plant signals exciting for theorists and ecologists alike.

The most obvious examples of honest plant signals are flowers that change colour once they have been pollinated (Figure 1f) [39]. These unrewarding flowers without nectar still contribute to attracting pollinators to as yet unpollinated flowers of the same plant from a long distance. Simultaneously, they also increase the foraging efficiency of pollinators once they arrive by directing them only to the rewarding flowers. This results in effective pollen transfer to as yet unpollinated flowers. Although floral colour can also indicate nectar quality and quantity (Figure 1g) [40], this correlation is expected to vary with the degree of specialization and plant density [5]. Because signals and rewards are uncoupled in flowers, we generally expect a high potential for dishonest signals, especially at the intraspecific level [41]. Indeed, any recently depleted flower offers a dishonest signal if its colour stays constant. Once a pollinator arrives at such a flower, it is likely to transfer pollen before it can assess the available nectar. Thus, from the perspective of the plant, pollinators are often 'tolerant' of flowers with variable rewards, reducing selection for honest signals.

Floral mimicry is a fascinating though little explored topic [42]. Roughly a third of all orchid species do not provide any reward to pollinators. Some such orchids are Batesian mimics, closely resembling a single rewarding model [43], whereas others broadly resemble several species with rewarding flowers [12]. The second strategy benefits from a behaviour called 'colour generalization', in which pollinators prefer novel colours that are most similar to those that they have learned to associate with rewards [11]. The evolutionary advantages of this strategy are lower production costs and a higher probability of outcrossing, because pollinators as less likely to visit several unrewarding flowers from the same plant $[41,44]$. Comparing the phylogenies of rewardless flowers across different taxa will help us to understand the occurrence of floral mimicry and the evolution of mimicry systems in general.

In fruits, the most obvious examples of dishonest signals are brightly coloured seeds, such as those of Margaritaria spp., that mimic fruits (Figure 1h). In other fruits, colour signals show a dichotomous pattern. In 45 species from Venezuela, red and black colouration was not associated with nutritive rewards, whereas blue, green, white and yellow-orange hues indicated protein, carbohydrate and tannin contents (Figure 1i) [8]. This pattern suggests two different signalling strategies: (i) conspicuous colours (red and black) to increase detectability; and (ii) less conspicuous colours (e.g. blue or green) to advertise specific contents to mutualists. In the second 
strategy, colour also correlates with tannins indicating simultaneously to fruit predators a well protected fruit [8]. Determining the extent to which rewards and defences are signalled honestly to mutualists and antagonists, respectively, is an exciting area of research in the unravelling of the evolution of signalling systems.

\section{Plant signals and sensory exploitation}

Signalling systems generally consist of four steps: (i) signal generation; (ii) transmission, (iii) reception; and (iv) perception [1]. The sensory drive model posits that all are interdependent, with changes in one inducing changes in the others [1,45]. Similarly, the different contexts (foraging or mate choice) in which signals are used are also interdependent. Accordingly, the perception of signals associated with foraging (i.e. of flowers or fruits) might have favoured the use of similarly coloured epigamic signals by fruit and flower consumers [46,47].

From an evolutionary perspective, the colours of fruits and flowers might have influenced the visual abilities of nectarivores and frugivores and vice versa [48]. For example, feeding on young red leaves or red fruits might underlie the evolution of trichromacy in primates [49-51]. Once trichromacy had arisen, intraspecific signals probably evolved to encompass more colours, given that trichromatic animals are neurologically tuned to respond to coloured signals. More specifically, colour vision of mammals, birds and insects that feed on fruits and flowers might have been tuned to detect efficiently the conspicuous colours of those resources. In a non-foraging context, signals using the same colours that the visual system evolved to detect most readily would be favoured because the tuning enables the receiver to detect the signal at a greater distance and because the receiver is already predisposed to react to such a signal. Once a preference for a certain signal evolved, it could then be recruited for other purposes (an example of exploitation of a preexisting bias).

The hypothesis that intraspecific signals in nectarivores and frugivores are derived through a pre-existing bias for colour signals from flowers and fruits is supported by the observation that colours used in male courtship displays of some birds and fish match the colours of their preferred fruits $[46,47]$. Likewise, primates consume mostly red and yellow fruits and often communicate with the opposite sex using red and yellow signals [52]. A rigorous test of the hypothesis, using phylogenetically controlled contrasts and controlling for different light habitats among a wide range of taxa, has yet to be attempted. Nevertheless, comparing plumage coloration in all passerine genera that commonly consume fruits and nectar with strictly insectivorous genera in a field guide of neotropical birds [53] shows a difference in plumage: genera feeding on fruits and nectar (e.g. tanagers and cotingas) are more often coloured red, yellow, blue and green on at least $30 \%$ of their body than they are coloured entirely brownish, greyish or blackish $\left(n=67, \chi^{2}=4.31\right.$, $\mathrm{df}=1, P<0.05)$. The opposite is true for insectivorous species $\left(n=219, \chi^{2}=143.0, \mathrm{df}=1, P<0.001\right)$. In butterflies, Costa Rican Nymphalidae subfamilies that commonly visit flowers (according to DeVries [54]) are more likely to sport red, orange, yellow and blue on the dorsal surface of their wings than are subfamilies that feed on rotting fruit, dung, fungi and other non-brightly coloured foods $\left(n=11, \chi^{2}=4.07, \mathrm{df}=1, P<0.05\right)$. Another form of exploiting pre-existing biases is to eavesdrop as an insect predator on the communication between flowers and bees. The crab spider Thomisus spectabilis exploits the innate preference of bees for contrasting flowers. The spider contrasts with its floral background and bees are more attracted to flowers at which the predator is present [55].

Plant colouration can affect animal signals more directly. Animals commonly deposit pigments from plant material into their ornaments. For example, fruit-eating cedar waxwings Bombycilla cedrorum deposit unmodified the red carotenoid pigment rhodoxanthin into their feathers [56]. Within the past 40 years, waxwings with orange instead of the normal yellow tail band have appeared in eastern North America. This change in feather colouration is paralleled by the expansion of an invasive fruit species containing the rhodoxanthin that is responsible for the orange feathers [56]. Consequently, the signals used by plants might directly translate into animal communication. Studying this relationship will yield insight into whether carotenoid pigments in male traits are honest indicators of immunocompetence and foraging abilities of those males, as is frequently argued in the literature on sexual selection (Box 1).

\section{Conclusion}

Signals abound in nature and signal theory has much to gain from embracing the diversity of signal types. For plant-animal communication, we have outlined perspectives that will advance our current understanding of what information is transmitted by visual signals (honesty), whom they address (multiple receivers), and how plant signals can impact the evolution of animal signalling (SENSORY EXPLOITATION; Box 2). One of the remaining challenges in understanding the evolution of visual signals is to determine how signals are processed by different types of receiver. Although realistic models of signal reception by retinal cells have been developed, our current knowledge is scant about how those signals are processed by the brain. However, models of colour perception are important for yielding predictions of signal diversity [57]. The complexity of perception is illustrated by pioneering work on signal processing in honeybees. Bees use different receptor types and different neural channels for colour and shape discrimination, depending on flower size and bee flight speed $[10,58]$. Consequently, a signal from the same flower can be perceived in different ways by the same bee, depending on its angle of perception and travel speed [10].

If the processes of perception within one receiver are variable, the variety is even bigger among multiple receivers of a signal. However, the influence that cognitive mechanisms have on signal perception is rarely compared interspecifically and signal processing is assumed to be equivalent across all species. For example, studies often analyse signal function according to the three colour components of human colour vision (hue, chroma and brightness) even though human colour vision is not 


\section{Box 1. Do plants signal immuno-active substances?}

The colours of fruits are usually assumed to relay general information about ripeness or nutritional reward $[8,67]$. However, the pigments that comprise those signals might themselves constitute rewards [68]. Carotenoids and anthocyanins, the two major types of pigment used for fruit colouration, can act as antioxidants, contributing to immunocompetence in consumers $[35,36]$. Carotenoid-based ornaments are also one of the main types of visual signal used in courtship. Given that carotenoids cannot be synthesized by animals, they must be ingested. A common but untested assumption is that carotenoids are scarce in the environment and therefore are used by researchers as a model for a condition-dependent trait, representing an honest advertisement of immunocompetence and/or superior foraging abilities $[35,36]$.

Quantifying the availability of carotenoids in fruits and the match between carotenoid and anthocyanin content and fruit signal will yield two important insights. First, if carotenoids in fruits are abundant, they probably do not function as a condition-dependent trait in fruit-eating birds, which by consuming fruits to meet nutritional requirements, might passively ingest a large amount of carotenoids. Second, if plants signal carotenoid contents, occasional consumers of fruit might

\section{Box 2. Future research perspectives}

Below, we outline important questions to be addressed by signal theory researchers.

\section{Signal design in plants}

- How strong is the phylogenetic influence on signal expression in fruits and flowers [48]? Assessing this question serves as a foundation to examine adaptive hypotheses of signal evolution.

- Flowers and fruits are often displayed against conspicuously coloured, non-green secondary structures, such as coloured infructescences and bracts $[19,62]$. Do these structures enhance detectability according to the eye of the receiver?

- Which signal components are evaluated most efficiently by different receiver groups, both mutualists and antagonists?

- Do fruit pigments constitute a reward that consumers actively seek [68]?

\section{Honest signalling}

- What are the costs of pigmentation in plants?

- Do flower and fruit signals commonly indicate the quality of the reward (e.g. nectar, oils, pollen or amino acids) $[8,40]$ ?

- Is signal colour a condition-dependent trait in plants? In other words, do signal components, such as colour intensity, vary with individual fitness of the sender?

- How common is mimicry in fruits and how closely do mimetic (reward less) signals resemble the rewarding model in flowers [9] and fruits?

\section{Multiple receivers}

- How do different receivers (mates and mutualists versus predators) contribute to the evolution of signals?

- Do signals match the cone sensitivities of intended receivers while being simultaneously relatively inconspicuous to unintended receivers?

- Are multiple receivers commonly responsible for maintaining polymorphism in plant signals [23]? If so, can the selective pressures of receiver groups be inferred from the frequencies of colour morphs in different populations?

\section{Sensory drive}

- How do foraging cues such as fruits and flowers, influence the intraspecific signalling systems of their consumers?

- How do neural processes of receivers interact with the signal design in different signalling systems?

- Do different signalling systems that address a common receiver interfere with each other? Are there, for example, interactions between brightly coloured aposematic insect signals and showy fruit colours as perceived by omnivorous birds [69]? feed on certain fruits to enhance their immunocompetence instead of ingesting fruits for energetic reasons. The first insight will shed a more discriminating light on the honesty of carotenoid signalling in animals, whereas the second might alter our current perspective of the role of secondary metabolites in plant-animal interactions.

Finally, viewing fruit pigments as rewards for frugivores provides an alternative hypothesis for why fruits are coloured as they are. Fruits (unlike flowers) are consumed by animal mutualists. Rather than matching the spectral sensitivities of frugivores, fruit colours might be determined by the colour of plant pigments (orange-red hues in carotenoids; red, purple, blue, and black hues in anthocyanins) that are physiologically important to seed dispersers. This hypothesis might provide more information about why black is such a common colour of fruits but not of flowers. Black hues in fruits are attributed to high concentrations of anthocyanins, which can increase 200-fold compared with red, pre-ripe stages [67]. If anthocyanin production entails costs, these might be balanced with the enhanced visitation of mutualists to fruits but not to flowers. However, fruit pigments might serve multiple functions (e.g. as antioxidants and sunscreens), making it difficult to examine the costs and benefits of pigment production.

representative of vision in other taxa [28]. The importance of using vision models $[13,59,60]$ to incorporate receptive and cognitive processes into the field of behavioural ecology is the most powerful lesson to learn from plantanimal communication. It will enable us to evaluate not only how receivers interact with signal design, but also which components of a signal are evaluated (and by whom) causing the signal to work efficiently.

\section{Acknowledgements}

We thank Thomas Emmel and Nick Haddad for sharing their insight on butterfly colouration. John Endler, Carlos Herrera, Silvia Lomáscolo, Daniel Osorio and Lars Chittka as well as two anonymous referees greatly improved earlier versions of the article. Tom Eisner, Martha Weiss and Claudia Gack kindly gave permission for using their images of plant signals in Figure 1. Daniel Osorio generously helped in the preparation of Figure 2.

\section{References}

1 Endler, J.A. and Basolo, A.L. (1998) Sensory ecology, receiver biases and sexual selection. Trends Ecol. Evol. 13, 415-420

2 Endler, J.A. (2000) Evolutionary implications of the interaction between animal signals and the environment. In Animal Signals (Espmark, Y. et al., eds), pp. 11-16, Tapir Academic Press

3 Endler, J.A. (1993) Some general comments on the evolution and design of animal communication systems. Philos. Trans. R. Soc. Lond. Ser. B 340, 215-225

4 Delhey, K. et al. (2003) Paternity analysis reveals opposing selection pressures on crown coloration in the blue tit (Parus caeruleus). Proc. R. Soc. Lond. Ser. B 270, 2057-2063

5 Gumbert, A. et al. (1999) Floral colour diversity in plant communities, bee colour space and a null model. Proc. R. Soc. Lond. Ser. B $266,1711-1716$

6 Schmidt, V. et al. (2004) Conspicuousness, not colour as foraging cue in plant-animal interactions. Oikos 106, 551-557

7 Bradshaw, H.D. and Schemske, W. (2003) Allele substitution at a flower colour locus produces a pollinator shift in monkeyflowers. Nature 426, 176-178

8 Schaefer, H.M. and Schmidt, V. (2004) Detectability and content as opposing signal characteristics in fruits. Proc. R. Soc. Lond. Ser. B 271 (Suppl.), S370-S373

9 Kunze, J. and Gumbert, A. (2001) The combined effect of color and odor on flower choice behavior of bumble bees in flower mimicry systems. Behav. Ecol. 12, 447-456

10 Spaethe, J. et al. (2001) Visual constraints in foraging bumblebees: flower size and color affect search time and flight behavior. Proc. Natl. Acad. Sci. U. S. A. 98, 3898-3903 
11 Gumbert, A. (2000) Color choices by bumble bees (Bombus terrestris): innate preferences and generalization after learning. Behav. Ecol. Sociobiol. 48, 36-43

12 Gumbert, A. and Kunze, J. (2001) Colour similarity to rewarding model plants affects pollination in a food deceptive orchid, Orchis boryi. Biol. J. Linn. Soc. 72, 419-433

13 Thery, M. and Casas, J. (2002) Predator and prey views of spider camouflage. Nature 415, 133

14 Andersson, S. and Dobson, H.E.M. (2003) Behavioral foraging responses by the butterfly Heliconius melpomene to Lantana camara floral scent. J. Chem. Ecol. 29, 2303-2318

15 Dobson, H. (1994) Floral volatiles in insect biology. In Insect-Plant Interactions (Bernays, E.A., ed.), pp. 47-81, CRC Press

16 Chittka, L. et al. (1999) Flower constancy, insect psychology, and plant evolution. Naturwissenschaften 86, 361-377

17 Harms, K.E. et al. (2000) Pervasive density-dependent recruitment enhances seedling diversity in a tropical forest. Nature 404, 493-495

18 Wheelwright, N.T. and Janson, C.H. (1985) Colors of fruit displays of bird dispersed plants in two tropical forests. Am. Nat. 126, 777-799

19 Borges, R.M. et al. (2003) Butterfly pollination and high-contrast visual signals in a low-density distylous plant. Oecologia 136, 571-573

20 Andersson, S. et al. (2002) Multiple receivers, multiple ornaments, and a trade-off between agonistic and epigamic signaling in a widowbird. Am. Nat. 160, 683-691

21 Endler, J.A. and Mappes, J. (2004) Predator mixes and the conspicuousness of aposematic signals. Am. Nat. 163, 532-547

22 Rowe, C. and Guilford, T. (2000) Aposematism: to be red or dead. Trends Ecol. Evol. 15, 261-262

23 Irwin, R.E. et al. (2003) The role of herbivores in the maintenance of a flower color polymorphism in wild radish. Ecology 84, 1733-1743

24 Medel, R. et al. (2003) Pollinator-mediated selection on the nectar guide phenotype in the Andean monkey flower, Mimulus luteus. Ecology 84, 1721-1732

25 Menzel, R. and Backhaus, W. (1991) Colour vision in insects. In Vision and Visual Dysfunction. VI. Perception of Colour (Gouras, P., ed.), pp. 262-293, Macmillan Press

26 Smith, V.C. and Pokorny, J. (1975) Spectral sensitivity of the foveal cone photopigments between 400 and $500 \mathrm{~nm}$. Vis. Res. 15, 161-171

27 Vorobyev, M. and Osorio, D. (1998) Receptor noise as a determinant of colour thresholds. Proc. R. Soc. Lond. Ser. B 265, 351-358

28 Bennett, A.T.D. et al. (1994) Sexual selection and the mismeasure of color. Am. Nat. 144, 848-860

29 Briscoe, A.D. and Chittka, L. (2001) The evolution of color vision in insects. Annu. Rev. Entomol. 46, 471-570

30 Wenny, D.G. and Levey, D.L. (1998) Directed seed dispersal by bellbirds in a tropical cloud forest. Proc. Natl. Acad. Sci. U. S. A. 95, 6204-6207

31 Irwin, R.E. et al. (2004) The dual role of floral traits: pollinator attraction and plant defense. Ecology 85, 1503-1511

32 Voigt, F.A. et al. A comparsion of morphological and chemical fruit traits between two sites with different frugivore assemblages. Oecologia (in press)

33 Gronquist, M. et al. (2001) Attractive and defensive functions of the ultraviolet pigments of a flower (Hypericum calycinum). Proc. Natl. Acad. Sci. U. S. A. 98, 13745-13750

34 Chittka, L. et al. (2001) Adaptation, constraint, and chance in the evolution of flower color and pollinator color vision. In Cognitive Ecology of Pollination (Chittka, L. and Thomson, J.D., eds), pp. 106-126, University Press

35 Faivre, B. et al. (2003) Immune activation rapidly mirrored in a secondary sexual trait. Science 300,103

36 Blount, J.D. et al. (2003) Carotenoid modulation of immune function and sexual attractiveness in zebra finches. Science 300, 125-127

37 Dale, J. (2000) Ornamental plumage does not signal male quality in red-billed queleas. Proc. $R$. Soc. Lond. Ser. B 267, 2143-2149

38 Grafen, A. (1990) Biological signals as handicaps. J. Theor. Biol. 144, 517-546

39 Weiss, M.R. (1991) Floral colour changes as cues for pollinators. Nature 354, 227-229

40 Melendez-Ackerman, E. (1997) Patterns of color and nectar variation across an Ipomopsis hybrid zone. Am. J. Bot. 84, 41-47
41 Thakar, J.D. et al. (2003) Nectarless flowers: ecological correlates and evolutionary stability. Oecologia 136, 565-570

42 Roy, B.A. and Widmer, A. (1999) Floral mimicry: a fascinating yet poorly understood phenomenon. Trends Plant Sci. 4, 325-330

43 Johnson, S.D. (2000) Batesian mimicry in the non-rewarding orchid Disapulchra, and its consequences for pollinator behaviour. Biol. J. Linn. Soc. 71, 119-132

44 Smithson, A. and Gigord, L.D.B. (2001) Are there fitness advantages in being a rewardless orchid? Reward supplementation experiments with Barlia robertiana. Proc. $R$. Soc. Lond. Ser. B 268, 1435-1441

45 Boughman, J.W. (2002) How sensory drive can promote speciation. Trends Ecol. Evol. 17, 571-577

46 Madden, J.R. and Tanner, K. (2003) Preferences for coloured bower decorations can be explained in a non-sexual context. Anim. Behav. $65,1077-1083$

47 Rodd, F.H. et al. (2001) A possible non-sexual origin of mate preference: are male guppies mimicking fruit? Proc. R. Soc. Lond. Ser. B 269, 475-481

48 Chittka, L. (1997) Bee color vision is optimal for coding flower color, but flower colors are not optimal for being coded - why? Israel J. Plant Sci. 45, 115-127

49 Regan, B.C. et al. (2001) Fruits, foliage and the evolution of primate colour vision. Philos. Trans. R. Soc. Lond. Ser. B 356, 229-283

50 Sumner, P. and Mollon, J.D. (2000) Catarrhine photopigments are optimised for detecting targets against a foliage background. J. Exp. Biol. 203, 1963-1986

51 Surridge, A.K. et al. (2003) Evolution and selection of trichromatic vision in primates. Trends Ecol. Evol. 18, 198-205

52 Dixson, A.F. (2000) Primate Sexuality, Oxford University Press

53 Ridgely, R.S. and Greenfield, P.J. (2001) The Birds of Ecuador, Christopher Helm

54 DeVries, P.J. (1987) The Butterflies of Costa Rica, Princeton University Press

55 Heiling, A.M. et al. (2003) Crab-spiders manipulate flower signals. Nature 421, 334

56 Witmer, M.C. (1996) Consequences of an alien shrub on the plumage coloration and ecology of cedar waxwings. Auk 113, 735-743

57 Kevan, P.G. et al. (1996) Why are there so many and so few white flowers? Trends Plant Sci. 1, 280-284

58 Hempel de Ibarra, N. and Giurfa, M. (2003) Discrimination of closed coloured shapes by honeybees requires only contrast to the long wavelength receptor type. Anim. Behav. 66, 903-910

59 Backhaus, W. (1991) Color opponent coding in the visual system of the honeybee. Vis. Res. 31, 1381-1397

60 Hart, N.S. et al. (1998) Visual pigments, oil droplets and cone photoreceptor distribution in the European starling (Sturnus vulgaris). J. Exp. Biol. 201, 3321-3328

61 Endler, J.A. and Thery, M. (1996) Interacting effects of lek placement, display behaviour, ambient light and colour patterns in three neotropical forest-dwelling birds. Am. Nat. 148, 421-452

62 Burns, K.C. and Dalen, J.L. (2002) Foliage color contrasts and adaptive fruit color variation in a bird-dispersed plant community. Oikos 96, 463-469

63 Kokko, H. et al. (2003) The evolution of mate choice and mating biases. Proc. R. Soc. Lond. Ser. B 270, 653-664

64 Ayasse, M. et al. (2000) Evolution of reproductive strategies in the sexually deceptive orchid Ophrys sphegodes: how does flower-specific variation of odor signals influence reproductive success? Evolution 54, 1995-2006

65 Knudsen, J.T. et al. (2001) Flower scent and pollination in selected neotropical palms. Plant Biol. 3, 642-653

66 Ayasse, M. et al. (2003) Pollinator attraction in a sexually deceptive orchid by means of unconventional chemicals. Proc. R. Soc. Lond. Ser. B 270, 517-522

67 Willson, M.F. and Whelan, C.J. (1990) The evolution of fruit color in fleshy-fruited plants. Am. Nat. 136, 790-809

68 Cipollini, M.L. (2000) Secondary metabolites of vertebrate-dispersed fruits: evidence for adaptive functions. Rev. Chil. Hist. Nat. 73, 421-440

69 Herrera, C.M. (1985) Aposematic insects as 6-legged fruits: incidental short-circuiting of their defense by frugivorous birds. Am. Nat. 126, 286-293 